\title{
Physiologie des Wochenhetts.
}

\section{Verhalten der Mutter.}

Das Wochenbett setzt sich aus 2 Vorgängen zusammen:

1) der Rückbildung der Genitalien,

2) der Bildung der Milch.

ad 1) Die Rü ckbildung der Genitalien ist nach 4 bis 6 Wochen beendet. Sie documentirt sieh hauptsächlich in der zunehmenden Verkleinerung des Uterus. und in der Veränderung des Lochialflusses. Während der Uterus am 1. Tag des Wochenbetts in Nabelhöhe steht, verschwindet er am 10. Tag hinter der Symphyse, am 5. Tag steht er ungeführ in der Mitte zwischen Nabel und Symphyse. Bis zum 10. Tag ist gewöhnlich noch der inmere Muttermund für einen Finger durchgängig, und die rauhe Placentastelle zu tühlen. Auffallend ist in dieser Zeit die starke Anteflexion des Uterus. Der Uterus verkleinert sich einmal durch Contractionen, "Nachwehen" schmerzhaft sind dieselben nur bei mp. - zweitens durch Verfettung und Resorption der Muskelprotoplasma.

Die Lochien sind das Produkt der wunden Uterusschleimhaut. Sie bestehen in den ersten 3 Tagen vornehmlich aus Blut (Lochia cruenta s. rubra), vom 4.-10. Tag aus Blut und Eiter - resp. Lymphkörperchen, Schleimkörperchen, Deciduazellen, Plattenepithelien und Mikroorganismen (Lochia sanguinolenta oder serosa - Heischwasserähnliches Aussehen), von da an versehwindet das Blut (Lochia alba). 4-6 Wochen nach der Geburt hört der Wochenfluss ganz auf. Normale Lochien riechen fade, aber sie stinken nicht! Bei Erstgebärenden besonders geht die Rückbildung oft langsamer vor sich, so dass der Uterus noch länger von aussen zu fühlen, und der Lochialfluss längor blntig gefärbt ist. Die neue Schleimhaut 
des Uterus bildet sich aus den zwischen den Muskeln liegenden Drüsenenden und dem sie umgebenden Bindegewebe.

ad 2. Die eigentliche $\mathrm{Milchsecretion} \mathrm{tritt} \mathrm{am} 3$. oder 4. Tage ein, indem die Brüste stark anschwellen, schmerzhaft werden und reichlich die fertige Milch entleeren. Dieselbe unterscheidet sich von dem Colostrum morphologisch durch den Zerfall der Drüsenzellen in feinste Fetttröpfchen, chemisch durch das Vorherrschen des Caseins gegenüber dem Albumin. Dass durch das sogenannte Einschiessen der Milch, also einen physiologischen Process, eine nach 24 Stunden wieder erscheinende fieberhafte Temperatursteigerung, das sogenannte Milchfieber hervorgerufen werden kann, wird neuerdings immer mehr angezweifelt, und jede Temperatursteigerung über $38^{\circ}$ als ein Krankheitssymptom betrachtet.

Die Milchsecretion tritt ganz unabhängig vom Anlegen auf, wird aber nur durch das Saugen unterhalten.

Sonstige auffallende Erscheinungen im Wochenbett: 1. Die Pulsverlangsamung (50-70 Schläge).

Sie erklärt sich nach $01 \mathrm{shausen}$ durch einen infolge der Fettresorption vom Uterus aus vermehrten Fettgehalt des Blutes.

2. Die Schweisssecretion. (Dieselbe soll nicht künstlich erzeugt werden).

3. Die Harn- und Stuhlverhaltung.

Dieselbe erklärt sich in manchen Fällen durch die mangelnde Action der Bauchpresse. In anderen Fällen beruht die Harnverhaltung auf einer Schwellung des Blasenbalses oder einer Knickung der Harnröhre. Endlich hält die Wöchnerin den Urin manchmal deswegen zurück, weil der die kleinen Fissuren im Vorhof benetzende Urin ihr brennende Schmerzen verursacht. Lässt man das Urinlassen im Liegen schon in der Schwangerschaft einüben resp. überrieselt man bei Fissuren während des Versuchs der Urinentleerung die Vulva mit abgekochtem Wasser, so sieht man viel seltener Urinverhaltung. Anm. Das zeitweise Harnträufeln der ersten Tage beruht auf einer Lähmung des Sphincter vesicae infolge stärkerer Quetschung, vom 5. Tage an auftretend, weist es auf eine Blasenscheidenfistel hin. 


\section{Das Verhalten des Neugeborenen.}

Die Kopfgeschwulst verschwindet rasch, das Cephalhaematom entsteht dagegen erst nach der Geburt. Dasselbe besteht in einem Bluterguss unter dem Periost. Es überschreitet daher nie die Nahtlinie. Es bedarf keiner Behandlung, da das Blut von sclbst wieder resorbirt wird. Innerhalb der ersten 24. Stunden muss das Kind Urin und Meconium entleeren. Ist dies nicht der Fall, so ist ein Verschluss der Harnröhre oder des Afters vorhanden. Da das Kind in den ersten Tagen hungert, so nimmt es c. $200 \mathrm{~g}$ an Gewicht ab, muss aber am 10. Tag das Anfangsgewicht wieder erreicht haben. Bei $80 \%$ der Kinder kommt es am 2,-3. 'Tag zu einem Icterus (neogonorum). Ein starker Icterus signalisirt schlechte Ernährungsverhältnisse (Runge). Der Icterus ad neogonum ist ein Symptom verschiedener Erkrankungen z. B. von Sepsis, Syphilis etc. Der Icterus neogonorum ist ein hepatogener. Beweis: das Vorhandensein von Gallensäuren in der Pericardialflüssigkeit icterischer Kinder. Der Nabelschnurrest fällt gewöhnlich am 5. Tag ab, die zurückbleibende Wunde ist am 12. Tag geheilt. Der Nabel kann der Ausgangspunkt einer tödtlichen septischen Erkrankung werden. Die Hebammen sind daher angewiesen, zunächst das Kind und dann die Mutter zu besorgen. Aus der physiologischen Milchsecretion der Neugeborenen entsteht eine Mastitis nur durch äussere Insulte.

\section{Die Diätetik des Wochenbetts.}

\section{Pflege der Wöchnerin.}

Die Wöchnerin muss solange das Bett hüten, als der Uterus von aussen zu fühlen ist (9-14 Tage). Frühes Aufstehen disponirt zum sogenannten Uterusvorfall.

In den ersten Tagen ist die Seitenlagerung verboten, damit keine Luft in den Uterus eintritt. Aufsitzen darf die Wöchnerin erst in der 2. Woche. Die lokale Behandlung beschränkt sich auf eine 2 mal täglich nach der Urinentleerung vorgenommenen Abspülung der änsseren Genitalien mit Sublimat 1: 5000 . Vor die Genitalien kommt ein Bausch Salicylwatte, der öfters ernenert wird. Die Unterlagen müssen mindestens 2 mal täglich 
gewechselt werden. Ist das spontane Uriniren unmöglich, so muss $2 \mathrm{mal}$ täglich catheterisirt werden. Dies geschieht mit gut desinficirter Hand nach der Abspülung der Genitalien. Die Urethralmündung wird dann noch mit einem in $3 \%$ ige Carbolsäurelösung getauchten Salicylwattebausch abgetupft. Der neusilberne Katheter wird vor dem Gebrauch ausgekocht, der Nela to n'sche wird in $0,3 \%$ iger Salicylsïurelösung auf bewalırt, nach dem Gebrauch mit Wasser gereinigt und für $1 / 4$ Stunde in Sublimat 1: 1000 gelegt. Wo der Arzt nicht sicher ist, dass die Wärterin diese Massregeln genau ausführt, eatheterisire er selbst. Durch Aufsteigen der Entzündung kanu eine Cystitis im Wochenbett zum Tode führen! Ist am 3. Tag noch kein Stuhlgang erfolgt, so gibt man einen Lı̈ffel Ricinns.

Die Diät der Wöchnerin muss eine leicht verdauliche aber nahrhafte sein, zumal wenn die Wöchnerin nährt. Jede gesunde Frau, die nicht von phthisischen Eltern stammt, soll ihr Kind selbst nähren! Im Allgemeinen wird durch das Nähren eine gute Rückbildung des Uterus begünstigt. Die Wiederkehr der Menstruation ist kein Grund, mit dem Stillen aufzuhören.

2 mal täglich ist die Temperatur der Wöchnerin zu messen!

Fordert keine specielle Indication dazu auf, so unterlässt man eine innere Untersuchung der Wöchnerin bis zu ihrer Entlassung aus der Behandlung.

\section{Pflege des Kindes.}

Die Hauptsache bei derselben ist zweckmässige Ernährung and äusserste Reinlichkeit. Ist die natürliche Ernährung nicht möglich, so gebe man im ersten Lebensjahr statt dessen gute, mit Wasser verđünnte und durch Milchzucker versüsste Kuhmilch, die man am besten in einem Soxhlet'schen Apparat abkochen lässt.

Unmittelbar nach der Geburt des Kopfes sind die Augen des Kindes, um die gonorrhoische Entzündung derselben zu verhindern, sorgfältig mit abgekochtem Wasser zu reinigen. Der Nabelschnurrest wird in ein trockenes Läppchen eingehüllt. Das Kind muss täglich gebadet werden (Temp. $28^{\prime \prime}$ R.). 
Vor jedem Anlegen muss es trocken gelegt, nach dem Anlegen muss $\mathrm{ihm}$ der Mund mit einem fenchten Läppchen ausge-

Fig. 10.

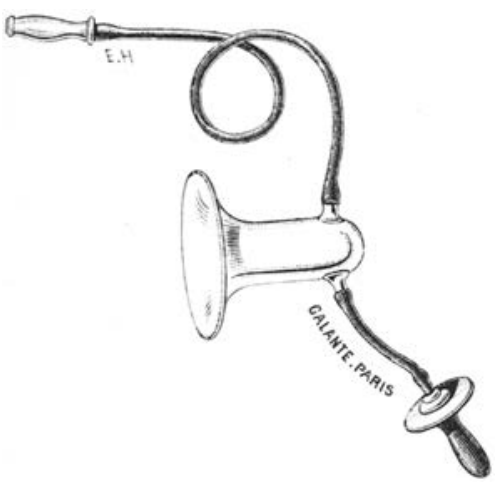
waschen werden. Auch die Warzen und ihre Umgebung müssen vor und nach dem Trinken mit vorher abgekochtem Wasser gereinigt werden. Sind die Warzen flach, oder ist gar eine Hohlwarze da, so dass das Kind die Warze nicht fassen kann, so lasse man ein Warzenhütchen aus Gummi oder ans Glas aufsetzen. (Fig. 10 stellt das von A u vard angegebene practische Warzenhütchen dar. An dem einen Pfropfen saugt die Mutter, an dem anderen das Kind.) Der Versuch muss erst in jedem Fall entscheiden, welcher Apparat die besten Dienste leistet.

Man gewöhne das Kind schon in der 2. Woche daran, dass es Nachts 6 Stunden pausirt. Am Tage wird es zunächst alle 2 Stunden, später alle 3 Stunden angelegt so dass es dann nur noch 6 Mahlzeiten zu sich nimmt. Das gesunde Kind soll am Ende des 4. Monats doppelt, am Ende des 12. Monats drei fach so schwer sein, wie bei der Geburt. Es soll ferner im 1. Monat $25 \mathrm{~g}$, im 12. Monat nur noch $6 \mathrm{~g}$ täglich zunehmen (Gerhardt). 